\title{
Time Reversal Space-Time Block Coding for FWA Systems
}

\author{
Pei Xiao, Rolando Carrasco \\ School of Electrical, Electronic and Computer Engineering \\ University of Newcastle, NE1 7RU, United Kingdom \\ E-mail: pei.xiao, r.carrasco@ncl.ac.uk \\ Ian Wassell \\ Lab for Communication Engineering, University of Cambridge \\ 15 JJ Thomson Avenue, CB3 0FD, United Kingdom \\ E-mail: ijw24@eng.cam.ac.uk
}

\begin{abstract}
The aim of this paper is to present the application of the time-reversal space-time coding (TR-STBC) on the broadband fixed wireless access (FWA) systems. In addition to the transmit diversity obtained from the TR-STBC scheme, we also consider the concatenation of TR-STBC and an outer channel code in order to provide coding gain for the FWA systems. A turbo equalization scheme is proposed for the concatenated systems. Different receiver strategies are compared, and their performance/complexity tradeoff is discussed.
\end{abstract}

\section{INTRODUCTION}

The rapid development of Internet services has increased the need for high-speed information exchange. The demands for data rates and quality of broadband services will increase dramatically in the near future. Comparing with the widely deployed cable and DSL, broadband fixed wireless access (FWA) has some advantages, which include avoiding distance limitations of DSL and high costs of cable, rapid deployment, high scalability, lower maintenance and upgrade costs, and granular investment to match market growth. Standardization of FWA systems is currently being undertaken by the IEEE 802.16 working group [1] and the ETSI HIPERMAN group [2]. Both orthogonal frequency division multiplexing (OFDM) and single-carrier solutions have been adopted in IEEE 802.16 standard as two alternatives for FWA systems operating at $2-11 \mathrm{GHz}$ bands [3]. An overview of FWA technology, the characteristics of FWA channels, and a description of the physical layer specifications can be found in $[4,5]$.

An effective solution for meeting the high data rate and high quality of service requirements is to use space-time coding (STC) techniques [6-8] which employ multiple antennas at both transmitter and receiver. They combine antenna array signal processing and coding technique, and provide significant capacity gains over traditional single antenna systems in wireless fading channels. Space-time codes were originally designed for flat fading channels and did not consider the intersymbol interference (ISI) introduced by frequency-selective channels. However, the assumption of flat-fading is not always justified, especially for wideband transmission, such as in the FWA systems where the channels exhibit frequency selectivity. It has been shown in [9] that direct implementation of STC on the FWA systems leads to unsatisfactory performance. In [10], the twoantenna transmit diversity scheme [7] proposed by Alamouti was generalized for frequency selective channels, where

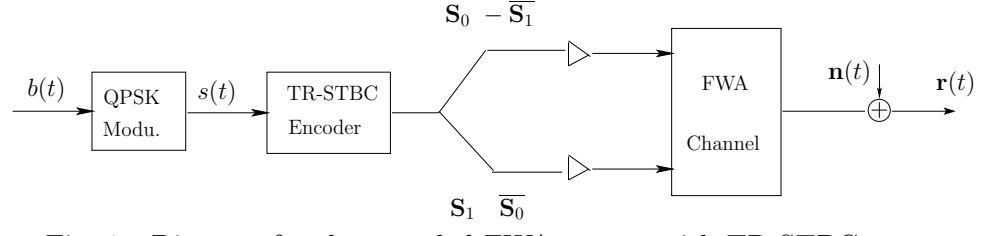

Fig. 1. Diagram for the uncoded FWA system with TR-STBC.

the transmitted signals are coded on a block-to-block basis rather than a symbol-by-symbol basis. This is so-called time-reversal (TR) STBC. In this paper, we study its performance in the FWA systems. To our best knowledge, the TR-STBC has not been considered for this application in the existing literature. Due to the quasi-static nature of the FWA channel, it is well suited for the TR-STBC application since the requirement for channel stationarity over a block of symbols can be readily satisfied. We introduce the TR-STBC to the uncoded FWA system in Section II and to the coded FWA system in Section III. In the latter case, a turbo equalization scheme which integrates equalization and channel decoding in an iterative fashion is proposed and compared with partitioned approach to equalization and decoding. Numerical results are shown in Section IV to demonstrate the performance of the discussed schemes. Finally, some conclusions are drawn in Section V.

\section{TR-STBC FOR UNCODED FWA SYSTEM}

Fig. 1 shows the baseband representation of the uncoded FWA system with TR-STBC under study. The information sequence $b(t)$ is mapped into QPSK symbol sequence $s(t)$. Unlike the Alamouti transmission scheme [7], which transmits two symbols from two antennas at a time, the TRSTBC encoder groups the symbols into two symbol blocks $\mathbf{S}_{0}$ and $\mathbf{S}_{1}$ at each antenna, each containing $N+1$ symbols. Two symbol blocks are simultaneously transmitted from two antennas. The data transmission is divided into two time frames. During the first time frame, $\mathbf{S}_{0}$ is transmitted from the first antenna; and $\mathbf{S}_{1}$ is transmitted from the second antenna. During the second transmission frame, the first antenna transmits a time reversed, complex conjugated and sign inverted version of $\mathbf{S}_{1}$, denoted as $-\overline{\mathbf{S}_{1}}$; the second antenna transmits a time reversed, complex conjugated version of $\mathbf{S}_{0}$, denoted as $\overline{\mathbf{S}_{0}}$. The transmission mechanism is illustrated in the upper diagram of Fig. 2. 


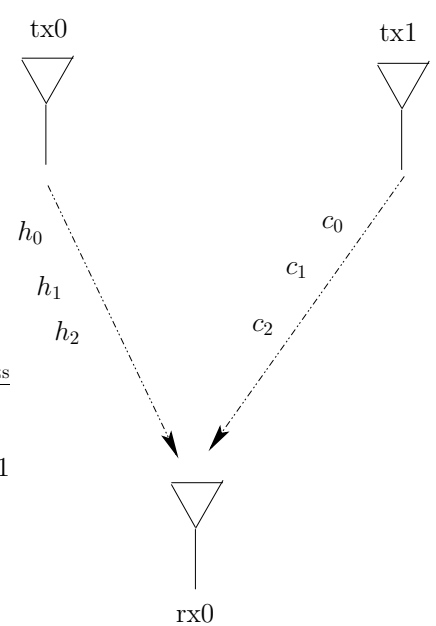

Fig. 3. Diagram for the 2TX-1RX SUI-3 channel.

The symbol streams are defined as follows:

$$
\begin{aligned}
\mathbf{S}_{0} & =\left\{s_{0}(0), s_{0}(1), \ldots, s_{0}(N)\right\} \\
\mathbf{S}_{1} & =\left\{s_{1}(0), s_{1}(1), \ldots, s_{1}(N)\right\} \\
\overline{\mathbf{S}_{0}} & =\left\{s_{0}^{*}(N), s_{0}^{*}(N-1), \ldots, s_{0}^{*}(0)\right\} \\
-\overline{\mathbf{S}_{1}} & =\left\{-s_{1}^{*}(N),-s_{1}^{*}(N-1), \ldots,-s_{1}^{*}(0)\right\}
\end{aligned}
$$

where ${ }^{*}$ denotes the conjugate transpose operation when applied to vectors and matrices, and simply the complex conjugate when applied to scalars.

The task of the receiver is to detect the transmitted information sequence $\mathbf{b}(t)$ given the received observation $\mathbf{r}(t)$. To this end, we need first to detect the transmitted QPSK symbol sequence $\mathbf{s}(t)$ which is corrupted with ISI and AWGN noise $\mathbf{n}(t)$.

Fig. 3 shows the FWA channels between the transmit and receive antennas. We consider the case when there are two transmit antennas and one receive antenna. Tailored for different terrain conditions, a set of 6 typical channel models, known as the Stanford University Interim (SUI) Channel Models have been proposed in [11] for simulation, design, development and testing of FWA systems. All of them are simulated using 3 taps, having either Rayleigh or Ricean amplitude distributions. For the purpose of this study, we select the SUI-3 channel which has 3 taps with a tap spacing of $500 \mathrm{~ns}$, and maximum tap delay of 1000ns. The channel between tx0 and $\mathrm{rx} 0$ can be represented by a discrete-time filter as $h\left(z^{-1}\right)=h_{0}+h_{1} z^{-1}+h_{2} z^{-2}$; the channel between tx1 and $\mathrm{rx} 0$ can be represented by a discrete-time filter as $c\left(z^{-1}\right)=c_{0}+c_{1} z^{-1}+c_{2} z^{-2}$. The channel coefficients are assume to remain constant during the transmission of one block of data,

The received signal during the first time frame can be expressed as

$$
\begin{aligned}
r_{0}(t)= & h\left(z^{-1}\right) s_{0}(t)+c\left(z^{-1}\right) s_{1}(t)+n_{0}(t) \\
= & h_{0} s_{0}(t)+h_{1} s_{0}(t-1)+h_{2} s_{0}(t-2) \\
& +c_{0} s_{1}(t)+c_{1} s_{1}(t-1)+c_{2} s_{1}(t-2)+n_{0}(t)
\end{aligned}
$$

where $t=0,1, \ldots, N$, and the noise $n_{0}(t) \sim \mathcal{C N}\left(0, N_{0}\right)$. The received signal during the second frame $r_{1}(t)$ is the time reversed and complex conjugated version of the origi- nal received signal $r_{1}^{\prime}(t)$, i.e., $r_{1}(t)=r_{1}^{\prime *}(N-t)$, or

$$
\begin{aligned}
r_{1}(t)= & c^{*}(z) s_{0}(t)-h^{*}(z) s_{1}(t)+n_{1}(t) \\
= & c_{0}^{*} s_{0}(t)+c_{1}^{*} s_{0}(t+1)+c_{2}^{*} s_{0}(t+2) \\
& -h_{0}^{*} s_{1}(t)-h_{1}^{*} s_{1}(t+1)-h_{2}^{*} s_{1}(t+2)+n_{1}(t)
\end{aligned}
$$

where $t=0,1, \ldots, N$, and the noise $n_{1}(t) \sim \mathcal{C N}\left(0, N_{0}\right)$. The formation of the received signal is illustrated in the lower diagram of Fig. 2. Equations (1) and (2) can be written in a compact form as

$$
\underbrace{\left[\begin{array}{c}
r_{0}(t) \\
r_{1}(t)
\end{array}\right]}_{\mathbf{r}(t)}=\underbrace{\left[\begin{array}{cc}
h\left(z^{-1}\right) & c\left(z^{-1}\right) \\
c^{*}(z) & -h^{*}(z)
\end{array}\right]}_{\mathbf{H}\left(z, z^{-1}\right)} \underbrace{\left[\begin{array}{c}
s_{0}(t) \\
s_{1}(t)
\end{array}\right]}_{\mathbf{s}(t)}+\underbrace{\left[\begin{array}{l}
n_{0}(t) \\
n_{1}(t)
\end{array}\right]}_{\mathbf{n}(t)}
$$

where

$$
\begin{aligned}
h\left(z^{-1}\right) & =h_{0}+h_{1} z^{-1}+h_{2} z^{-2} \\
-h^{*}(z) & =-h_{0}^{*}-h_{1}^{*} z-h_{2}^{*} z^{2} \\
c\left(z^{-1}\right) & =c_{0}+c_{1} z^{-1}+c_{2} z^{-2} \\
c^{*}(z) & =c_{0}^{*}+c_{1}^{*} z+c_{2}^{*} z^{2}
\end{aligned}
$$

The channel matrix $\mathbf{H}\left(z, z^{-1}\right)$ is orthogonal since

$$
\begin{aligned}
\mathbf{H}^{*}\left(z, z^{-1}\right) \mathbf{H}\left(z, z^{-1}\right) & =\left[\begin{array}{cc}
h^{*}(z) & c\left(z^{-1}\right) \\
c^{*}(z) & -h\left(z^{-1}\right)
\end{array}\right]\left[\begin{array}{cc}
h\left(z^{-1}\right) & c\left(z^{-1}\right) \\
c^{*}(z) & -h^{*}(z)
\end{array}\right] \\
& =\left[h^{*}(z) h\left(z^{-1}\right)+c^{*}(z) c\left(z^{-1}\right)\right] \mathbf{I} \\
& =\gamma\left(z, z^{-1}\right) \mathbf{I}
\end{aligned}
$$

where

$$
\begin{aligned}
\gamma\left(z, z^{-1}\right)= & \left(h_{0}^{*}+h_{1}^{*} z+h_{2}^{*} z^{2}\right)\left(h_{0}+h_{1} z^{-1}+h_{2}^{-2}\right) \\
& +\left(c_{0}^{*}+c_{1}^{*} z+c_{2}^{*} z^{2}\right)\left(c_{0}+c_{1} z^{-1}+c_{2}^{-2}\right) \\
= & \gamma_{0}+\gamma_{1} z+\gamma_{2} z^{2}+\gamma_{1}^{\prime} z^{-1}+\gamma_{2}^{\prime} z^{-2}
\end{aligned}
$$

and

$$
\begin{aligned}
\gamma_{0} & =\left|h_{0}\right|^{2}+\left|h_{1}\right|^{2}+\left|h_{2}\right|^{2}+\left|c_{0}\right|^{2}+\left|c_{1}\right|^{2}+\left|c_{2}\right|^{2} \\
\gamma_{1} & =h_{0} h_{1}^{*}+h_{1} h_{2}^{*}+c_{0} c_{1}^{*}+c_{1} c_{2}^{*} \\
\gamma_{2} & =h_{0} h_{2}^{*}+c_{0} c_{2}^{*} \\
\gamma_{1}^{\prime} & =h_{1} h_{0}^{*}+h_{2} h_{1}^{*}+c_{1} c_{0}^{*}+c_{2} c_{1}^{*}=\gamma_{1}^{*} \\
\gamma_{2}^{\prime} & =h_{2} h_{0}^{*}+c_{2} c_{0}^{*}=\gamma_{2}^{*}
\end{aligned}
$$

In the receiver, the signal vector $\mathbf{r}(t)$ is filtered by the matched filter $\mathbf{H}^{*}\left(z, z^{-1}\right)$, the output of which is given by

$$
\mathbf{z}(t)=\mathbf{H}^{*}\left(z, z^{-1}\right) \mathbf{r}(t)=\left[\begin{array}{cc}
h^{*}(z) & c\left(z^{-1}\right) \\
c^{*}(z) & -h\left(z^{-1}\right)
\end{array}\right]\left[\begin{array}{l}
z_{0}(t) \\
z_{1}(t)
\end{array}\right]
$$

or

$$
\begin{aligned}
z_{0}(t)= & h^{*}(z) r_{0}(t)+c\left(z^{-1}\right) r_{1}(t) \\
= & h_{0}^{*} r_{0}(t)+h_{1}^{*} r_{0}(t+1)+h_{2}^{*} r_{0}(t+2) \\
& +c_{0} r_{1}(t)+c_{1} r_{1}(t-1)+c_{2} r_{1}(t-2) \\
z_{1}(t)= & c^{*}(z) r_{0}(t)-h\left(z^{-1}\right) r_{1}(t) \\
= & c_{0}^{*} r_{0}(t)+c_{1}^{*} r_{0}(t+1)+c_{2}^{*} r_{0}(t+2) \\
- & h_{0} r_{1}(t)-h_{1} r_{1}(t-1)-h_{2} r_{1}(t-2)
\end{aligned}
$$




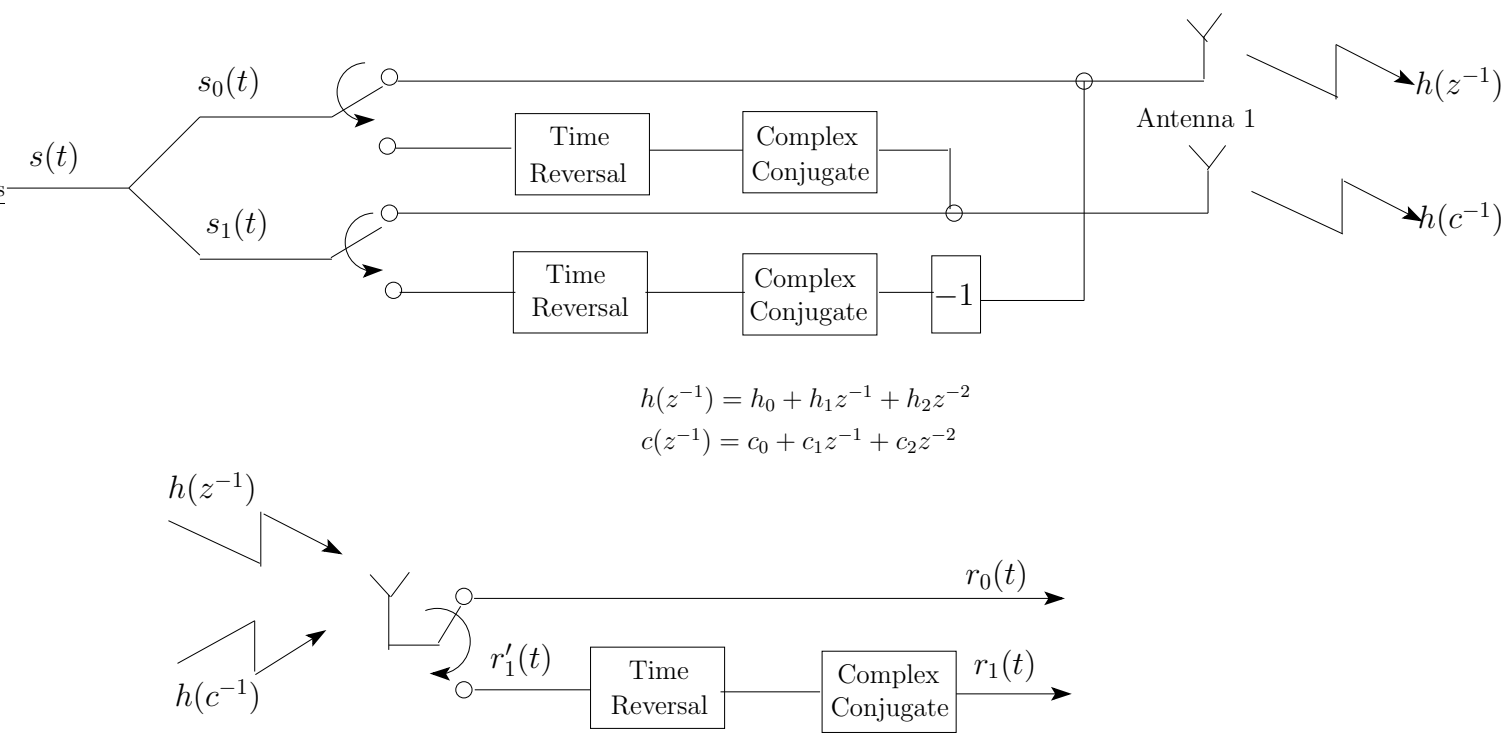

Fig. 2. Time-reversal STBC encoder and receiver front end [10].

The problem of detecting the symbol streams $s_{0}(t)$ and $s_{1}(t)$ are now decoupled since

$$
\begin{aligned}
\mathbf{z}(t) & =\mathbf{H}^{*}\left(z, z^{-1}\right) \mathbf{r}(t)=\mathbf{H}^{*}\left(z, z^{-1}\right)\left[\mathbf{H}\left(z, z^{-1}\right) \mathbf{s}(t)+\mathbf{n}(t)\right] \\
& =\mathbf{H}^{*}\left(z, z^{-1}\right) \mathbf{H}\left(z, z^{-1}\right) \mathbf{s}(t)+\mathbf{H}^{*}\left(z, z^{-1}\right) \mathbf{n}(t) \\
& =\gamma\left(z, z^{-1}\right) \mathbf{I} \cdot \mathbf{s}(t)+\mathbf{v}(t)
\end{aligned}
$$

where $\gamma\left(z, z^{-1}\right)=\gamma_{0}+\gamma_{1} z+\gamma_{2} z^{2}+\gamma_{1}^{\prime} z^{-1}+\gamma_{2}^{\prime} z^{-2}$. The matched filter output can be expressed alternatively as

$$
\left[\begin{array}{l}
z_{0}(t) \\
z_{1}(t)
\end{array}\right]=\gamma\left(z, z^{-1}\right) \mathbf{I}\left[\begin{array}{l}
s_{0}(t) \\
s_{1}(t)
\end{array}\right]+\left[\begin{array}{l}
v_{0}(t) \\
v_{1}(t)
\end{array}\right]
$$

Apparently, the TR-STBC converts the $L$-path multipleinput, multiple-output (MIMO) channels into $(2 L-1)$-path single-input, single-output (SISO) channels. The effective channel gain for desired signal is

$$
\gamma_{0}=\left|h_{0}\right|^{2}+\left|h_{1}\right|^{2}+\left|h_{2}\right|^{2}+\left|c_{0}\right|^{2}+\left|c_{1}\right|^{2}+\left|c_{2}\right|^{2},
$$

which is the total gain from different channels' different paths. From (5), we see that both spatial and temporal diversities are fully exploited by employing the TR-STBC scheme, and the desired signal is greatly enhanced. The filtered noise sequence vector $\mathbf{v}(t)=\left[v_{0}(t) v_{1}(t)\right]^{T}$ (where $T$ denotes the transpose operation) is still white since the autocorrelation matrix of $\mathbf{v}(t)$ is

$$
\begin{aligned}
& R_{\mathbf{v v}}\left(z, z^{-1}\right)=\mathrm{E}\left[\mathbf{v}(t) \mathbf{v}^{*}(t)\right] \\
& =\mathrm{E}\left[\mathbf{H}^{*}\left(z, z^{-1}\right) \mathbf{n}(t) \mathbf{n}^{*}(t) \mathbf{H}\left(z, z^{-1}\right)\right] \\
& =\mathbf{H}^{*}\left(z, z^{-1}\right) \mathrm{E}\left[\mathbf{n}(t) \mathbf{n}^{*}(t)\right] \mathbf{H}\left(z, z^{-1}\right)=\gamma\left(z, z^{-1}\right) N_{0} \mathbf{I}
\end{aligned}
$$

In the above equation, we use the fact that $\mathbf{n}(t)$ is a white noise sequence vector with $R_{\mathbf{n n}}\left(z, z^{-1}\right)=N_{0} \mathbf{I}$. The filtered noise $v_{0}(t)$ and $v_{1}(t)$ are uncorrelated, the two symbol streams $z_{0}(t)$ and $z_{1}(t)$ can therefore be decoded separately. The filtered noise variance $N_{v}$ can be derived as

$$
\begin{aligned}
& v_{0}(t)=h^{*}(z) n_{0}(t)+c\left(z^{-1}\right) n_{1}(t)=h_{0}^{*} n_{0}(t)+h_{1}^{*} n_{0}(t+1) \\
& \quad+h_{2}^{*} n_{0}(t+2)+c_{0} n_{1}(t)+c_{1} n_{1}(t-1)+c_{2} n_{1}(t-2) \\
& N_{v}=\mathrm{E}\left[v_{0}(t) v_{0}^{*}(t)\right]=\gamma_{0} N_{0}
\end{aligned}
$$

The TR-STBC itself only decouples the symbol streams from two transmit antennas. It, however, does not resolve the ISI in each symbol stream as we can see from (4). The ISI of course still needs to be handled by an equalizer. For example, we can apply a linear equalizer (LE) designed under minimum mean square (MMSE) criterion to the decoupled symbol streams $z_{0}(t), z_{1}(t)$ before making a decision on the transmitted symbols and bits.

\section{TR-STBC FOR CODED FWA SYSTEM}

It is well-known that STBCs, as well as TR-STBCs are able to exploit transmit diversity, but fail to provide any coding gain. To work around this problem, one can use space-time trellis code (STTC) which is an extension of trellis coded modulation to the systems with multiple antennas. It provides both full diversity and coding gain. However, it has high decoding complexity which grows exponentially with the number of antennas. Another option is to concatenate the STBC with an outer channel code, which is the approach we take here. In this section, we study the FWA system employing channel coding and develop a turbo equalization scheme which integrates the equalization and channel decoding in an iterative manner. Fig. 4 shows the block diagram for the coded FWA system. The information sequence $\left\{b_{n}\right\}$ is convolutionally encoded into coded bits $\left\{u_{n}\right\}$, which are subsequently interleaved and each block of two coded and interleaved bits $u_{n}^{\prime}[0], u_{n}^{\prime}[1]$ is mapped into one of the four QPSK symbols $s_{n}=x_{n}^{0}+j x_{n}^{1}$. The symbols are TR-STBC encoded according to the mechanism specified in Section II. The received signal is basically the same as expressed in (4) except the QPSK symbols are now formed by coded bits rather than information bits. The interleaver and deinterleaver are denoted as $\Pi$ and $\Pi^{-1}$, in Fig. 4, Fig. 5, and Fig. 6, respectively.

Two different receiver strategies are studied in this section. The first one is the partitioned equalization and decoding. In the receiver, the signal vector $\mathbf{r}(t)$ is first filtered by the matched filter $\mathbf{H}^{*}\left(z, z^{-1}\right)$. We consider two scenarios 3 for this partitioned approach. In the first scenario as shown 


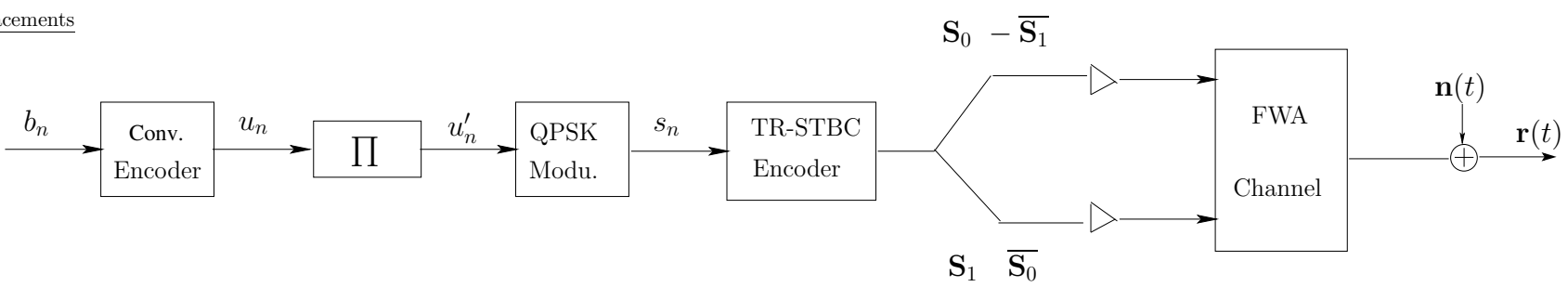

Fig. 4. Diagram for the FWA system with TR-STBC and channel coding.

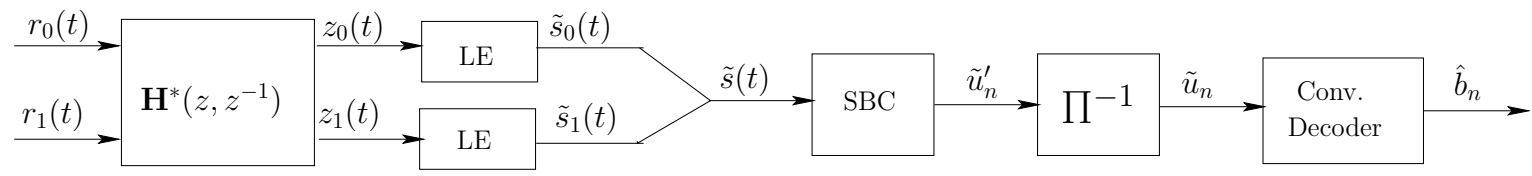

Fig. 5. Partitioned equalization and decoding algorithm for the FWA system.

in Fig. 5, the decoupled symbol streams from the matched filter $z_{0}(t)$ and $z_{1}(t)$ are each processed by a linear equalizer (LE) to derive an estimate of the transmitted symbol sequences $\tilde{s}_{0}(t)$ and $\tilde{s}_{1}(t)$, which are combined into a single symbol sequence. Then we perform symbol-to-bit conversion (SBC), deinterleaving and decoding to obtain an estimate of transmitted information bits $\left\{\hat{b}_{n}\right\}$. The second scenario is similar to the first one except that the equalization block is skipped. We either make hard decision on the transmitted symbols based on the matched filter output $z_{0}(t)$ and $z_{1}(t)$, followed by symbol-to-bit conversion to yield the hard decision on the coded bits, which are subsequently deinterleaved and decoded using hard-decision decoding; or we can pass the soft output $z_{0}(t)$ and $z_{1}(t)$ directly to the SBC to yield soft decision on the coded bits, which are then deinterleaved and decoded using soft-decision decoding.

Fig. 6 shows an integrated approach to equalization and decoding. In contrast to the previous partitioned strategy, the two blocks are performed jointly in an iterative manner. First, we use the matched filter $\mathbf{H}^{*}\left(z, z^{-1}\right)$ and LE to derive an initial estimate of the transmitted symbol sequences $\hat{s}_{0}(t)$ and $\hat{s}_{1}(t)$ (i.e., the estimate of $\mathbf{S}_{0}$ and $\mathbf{S}_{1}$ ). Each of the symbol stream is passed to an interference canceler (IC), and the ISI canceled version of the filtered signal is mapped to the log-likelihood ratio (LLR) of the coded bits, denoted as $\lambda\left(u_{n 0}^{\prime} ; O\right)$ and $\lambda\left(u_{n 1}^{\prime} ; O\right)$ for the first and second branch (stream), respectively. The LLRs from two branches are combined and deinterleaved to yield $\lambda\left(u_{n}^{\prime} ; I\right)$ as input to the Log-MAP decoder. We use the notations $\lambda(\cdot ; I)$ and $\lambda(\cdot ; O)$ to denote the input and output ports of a soft-input and soft-output device. Based on the soft inputs, a LogMAP decoder computes the LLR for each information bit $\lambda\left(b_{n} ; O\right)$ and each coded bit $\lambda\left(u_{n} ; O\right)$. The former is used to make decisions on the transmitted information bit at the final iteration, and $\lambda\left(u_{n} ; O\right)$ is interleaved and split into two streams of LLRs $\lambda\left(u_{n 0}^{\prime} ; I\right)$ and $\lambda\left(u_{n 1}^{\prime} ; I\right)$. Each stream is passed through a bit-to-symbol converter (BSC) to derive a soft symbol estimate $\lambda\left(s_{n}\right)=\lambda\left(x_{n}^{0}+j x_{n}^{1}\right)$, which is used for equalization at the next iteration. The derivation of $\lambda\left(u_{n}^{\prime} ; O\right)$ at the output of the IC \& Mapper block in Fig. 6 will be explained in detail next.

We focus on how the matched filter output $z_{0}(t)$ is processed to obtain the LLRs $\lambda\left(u_{n 0}^{\prime} ; O\right)$. Another output $z_{1}(t)$ can be processed in the exactly the same way to obtain the LLRs $\lambda\left(u_{n 1}^{\prime} ; O\right)$. To simplify the notations, we denote $z_{n}=z_{0}[n], s_{n}=s_{0}[n], v_{n}=v_{0}[n]$. Based on (4), the signal $z_{0}(t)$ can be written in vector form as $\mathbf{z}_{n}=\Gamma \mathbf{s}_{n}+$ $\mathbf{v}_{n}$, where $\mathbf{z}_{n}=\left[\begin{array}{lllll}z_{n-2} & z_{n-1} & z_{n} & z_{n+1} & z_{n+2}\end{array}\right]^{T}, \mathbf{s}_{n}=$ $\left[\begin{array}{lllllllll}s_{n-4} & s_{n-3} & s_{n-2} & s_{n-1} & s_{n} & s_{n+1} & s_{n+2} & s_{n+3} & s_{n+4}\end{array}\right]^{T}$, $\mathbf{v}_{n}=\left[\begin{array}{lllll}v_{n-1} & v_{n-2} & v_{n} & v_{n+1} & v_{n+2}\end{array}\right]^{T}$, and

$$
\boldsymbol{\Gamma}=\left[\begin{array}{ccccccccc}
\gamma_{2}^{\prime} & \gamma_{1}^{\prime} & \gamma_{0} & \gamma_{1} & \gamma_{2} & 0 & 0 & 0 & 0 \\
0 & \gamma_{2}^{\prime} & \gamma_{1}^{\prime} & \gamma_{0} & \gamma_{1} & \gamma_{2} & 0 & 0 & 0 \\
0 & 0 & \gamma_{2}^{\prime} & \gamma_{1}^{\prime} & \gamma_{0} & \gamma_{1} & \gamma_{2} & 0 & 0 \\
0 & 0 & 0 & \gamma_{2}^{\prime} & \gamma_{1}^{\prime} & \gamma_{0} & \gamma_{1} & \gamma_{2} & 0 \\
0 & 0 & 0 & 0 & \gamma_{2}^{\prime} & \gamma_{1}^{\prime} & \gamma_{0} & \gamma_{1} & \gamma_{2}
\end{array}\right]
$$

The interference canceled version of the received vector is given as

$$
\mathbf{z}_{n}^{\prime}=\mathbf{z}_{n}-\boldsymbol{\Gamma} \overline{\mathbf{s}}_{n}=\boldsymbol{\Gamma}\left[\mathbf{s}_{n}-\overline{\mathbf{s}}_{n}\right]+\mathbf{v}_{n}=\mathbf{h} s_{n}+\mathbf{w}_{n}
$$

where $\mathbf{h}=\left[\begin{array}{lllll}\gamma_{2} & \gamma_{1} & \gamma_{0} & \gamma_{1}^{\prime} & \gamma_{2}^{\prime}\end{array}\right]^{T}$ is the channel vector, and $\mathbf{w}_{n}=\left[\begin{array}{lllll}w_{n-2} & w_{n-1} & w_{n} & w_{n+1} & w_{n+2}\end{array}\right]^{T}$ stands for the combined noise and interference cancellation residual vector. We denote $\bar{s}_{n-i}$ as a soft estimate of $s_{n-i}$ from previous stage, and $\left\{\bar{s}_{n-i}=\bar{x}_{n-i}^{0}+j \bar{x}_{n-i}^{1}\right\}$ is computed according to its LLR value as

$$
\bar{s}_{n-i}=\tanh \left[\lambda\left(x_{n-i}^{0}\right) / 2\right] / \sqrt{2}+j \tanh \left[\lambda\left(x_{n-i}^{1}\right) / 2\right] / \sqrt{2}
$$

To simplify the notation, the iteration (stage) index is omitted whenever no ambiguity arises. The vector $\overline{\mathbf{s}}_{n}=$ $\left[\begin{array}{lllllll}\bar{s}_{n-4} & \ldots & \bar{s}_{n-1} & 0 & \bar{s}_{n+1} & \ldots & \bar{s}_{n+4}\end{array}\right]^{T}$ contains the soft estimate of the interference symbols from the previous iteration.

Next, we shall demonstrate how the LLRs can be computed directly from the interference canceled signal. In case of perfect cancellation $\mathbf{w}_{n}=\mathbf{v}_{n}, \quad N_{w}=N_{v}=\gamma_{0} N_{0}$, according to (6). The conditional pdf of $\mathbf{z}_{n}^{\prime}$ is thus derived as

$$
f\left(\mathbf{z}_{n}^{\prime} \mid s_{m}\right)=\frac{1}{\left(\pi \gamma_{0} N_{0}\right)^{5}} \exp \left(-\frac{\left\|\mathbf{z}_{n}^{\prime}-\mathbf{h} s_{m}\right\|^{2}}{\gamma_{0} N_{0}}\right)
$$

For the QPSK modulated signals, the symbol LLR $\lambda\left(s_{n}\right)=\lambda\left(x_{n}^{0}\right)+j \lambda\left(x_{n}^{1}\right)$ to bits LLRs $\lambda\left(u_{n 0}^{\prime}[0]\right), \lambda\left(u_{n 0}^{\prime}[1]\right)$ mapping rule is simply $\lambda\left(u_{n 0}^{\prime}[0] ; O\right)=\lambda\left(x_{n}^{0}\right) ; \quad \lambda\left(u_{n 0}^{\prime}[1] ; O\right)=$ $\lambda\left(x_{n}^{1}\right)$. Next, we show how the LLR value of $x_{n}^{0}$ and $x_{n}^{1}$ can 


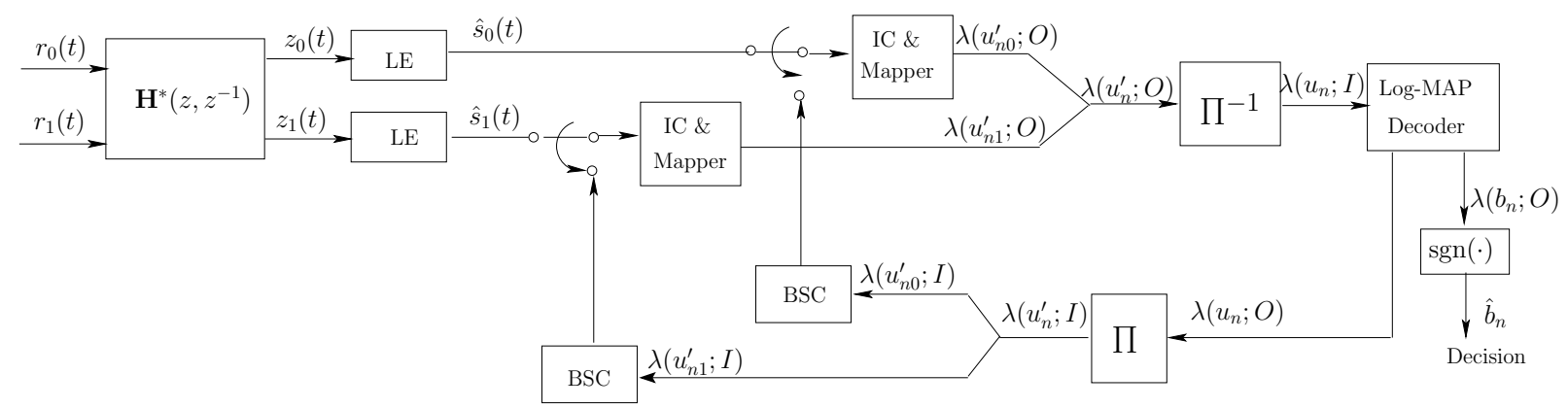

Fig. 6. Turbo equalization for the FWA system.

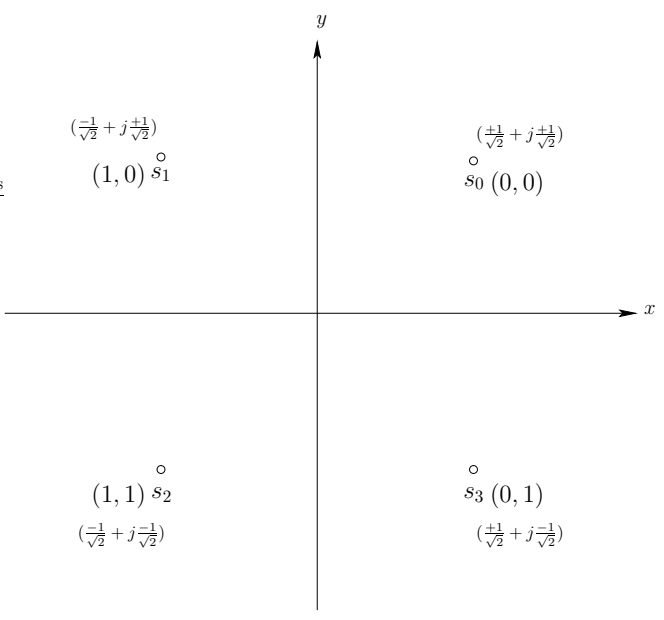

Fig. 7. QPSK constellation and bit-to-symbol mapping.

be derived from $\mathbf{z}_{n}^{\prime}$. Based on (7), the LLR of $x_{n}^{0}$ can be computed as

$$
\begin{aligned}
\lambda\left(x_{n}^{0}\right) & =\ln \frac{f\left(\mathbf{z}_{n}^{\prime} \mid x_{n}=0\right)}{f\left(\mathbf{z}_{n}^{\prime} \mid x_{n}=1\right)}=\ln \frac{f\left(\mathbf{z}_{n}^{\prime} \mid s_{0}\right)+f\left(\mathbf{z}_{n}^{\prime} \mid s_{3}\right)}{f\left(\mathbf{z}_{n}^{\prime} \mid s_{1}\right)+f\left(\mathbf{z}_{n}^{\prime} \mid s_{2}\right)} \\
& \approx \ln \frac{\exp \left(-\left\|\mathbf{z}_{n}^{\prime}-\mathbf{h} s_{+}\right\|^{2} /\left(\gamma_{0} N_{0}\right)\right)}{\exp \left(-\left\|\mathbf{z}_{n}^{\prime}-\mathbf{h} s_{-}\right\|^{2} /\left(\gamma_{0} N_{0}\right)\right)} \\
& =\frac{1}{\gamma_{0} N_{0}}\left\{\left\|\mathbf{z}_{n}^{\prime}-\mathbf{h} s_{-}\right\|^{2}-\left\|\mathbf{z}_{n}^{\prime}-\mathbf{h} s_{+}\right\|^{2}\right\} \\
& =\frac{2}{\gamma_{0} N_{0}} \operatorname{Re}\left\{\left(\mathbf{h} s_{+}\right)^{*} \mathbf{z}_{n}^{\prime}-\left(\mathbf{h} s_{-}\right)^{*} \mathbf{z}_{n}^{\prime}\right\}
\end{aligned}
$$

where $s_{+}$denotes the QPSK symbol corresponding to $\max \left\{f\left(\mathbf{z}_{n}^{\prime} \mid s_{0}\right), f\left(\mathbf{z}_{n}^{\prime} \mid s_{3}\right)\right\}$, and $s_{-}$denotes the QPSK symbol corresponding to $\max \left\{f\left(\mathbf{z}_{n}^{\prime} \mid s_{1}\right), f\left(\mathbf{z}_{n}^{\prime} \mid s_{2}\right)\right\}$ since the real part of the symbols $s_{0}, s_{3}$ corresponds to 0 , and the real part of the symbols $s_{1}, s_{2}$ corresponds to 1 as shown in Fig. 7 . Dual maxima rule [12] is used in (8) utilizing the fact that one term usually dominates each sum. Similarly,

$$
\begin{aligned}
\lambda\left(x_{n}^{1}\right) & =\ln \frac{f\left(\mathbf{z}_{n}^{\prime} \mid s_{0}\right)+f\left(\mathbf{z}_{n}^{\prime} \mid s_{1}\right)}{f\left(\mathbf{z}_{n}^{\prime} \mid s_{2}\right)+f\left(\mathbf{z}_{n}^{\prime} \mid s_{3}\right)} \\
& \approx \frac{2}{\gamma_{0} N_{0}} \operatorname{Re}\left\{\left(\mathbf{h} s_{+}\right)^{*} \mathbf{z}_{n}^{\prime}-\left(\mathbf{h} s_{-}\right)^{*} \mathbf{z}_{n}^{\prime}\right\}
\end{aligned}
$$

where $s_{+}$denotes the QPSK symbol corresponding to $\max \left\{f\left(\mathbf{z}_{n}^{\prime} \mid s_{0}\right), f\left(\mathbf{z}_{n}^{\prime} \mid s_{1}\right)\right\}$, and $s_{-}$denotes the QPSK symbol corresponding to $\max \left\{f\left(\mathbf{z}_{n}^{\prime} \mid s_{2}\right), f\left(\mathbf{z}_{n}^{\prime} \mid s_{3}\right)\right\}$ since the imaginary part of the symbols $s_{0}, s_{1}$ corresponds to 0 , and the imaginary part of the symbols $s_{2}, s_{3}$ corresponds to 1 as shown in Fig. 7.
Due to the assumption of perfect cancellation in the derivation of LLRs, this scheme is suboptimum during the initial stages of turbo equalization, but will approach optimality when the ISI is effectively canceled as the iterative process proceeds.

\section{NumERICAL RESULTS}

Numerical results are presented in this section to assess the performance of the discussed algorithms. For the coded system, we employ a rate 1/3 Maximum Free Distance convolutional code with constraint length 5 and generator polynomials $(25,33,37)$ in octal form. During each Monte-Carlo run, the block size is set to 1996 information bits followed by 4 tails bits to terminate the trellis, which corresponds to $2000 \times 3=6000$ coded bits or 3000 QPSK symbols, 200 of which are used as pilots to acquire a channel estimate. Channel estimation is conducted with the minimum variance unbiased estimator introduced in [13]. The coded bits are interleaved by a random interleaver. QPSK symbols are transmitted over the SUI-3 FWA channels. The channel coefficients vary from one block to another, however, they are assumed to remain constant during the transmission of one block of data. The antenna correlation coefficient is set to 0.4. The noise variance $N_{0}$ and path delays are assumed to be known to the receiver. The simulation curves are obtained by averaging the simulation results over at least 500 channel realizations. To study the behavior of turbo equalization algorithm presented in Section III, the number of iterations is set to 3 since it is observed that no more than 3 stages are needed for the system to converge.

Fig. 8 shows the performance of the TR-STBC for the uncoded FWA system. Apparently, the uncoded system cannot operate without equalization since the TR-STBC only converts MIMO channels into SISO channels. The ISI has to be handled by a SISO equalizer. To this end, we apply a MMSE linear equalizer with different lengths. As indicated by the plot, longer length results in a better performance. However, the gain becomes negligible when the equalizer length goes beyond 9 taps.

Fig. 9 shows the performance of the TR-STBC for the coded FWA system. In contrast to the uncoded system, it is possible for the coded FWA system to operate without equalization when the soft output from the matched filter is used for decoding (the performance is worst in the case of hard decision decoding). The rationale is that the TRSTBC exploits both spatial and temporal diversities, the desired signal is greatly enhanced. In the meantime, the effect 5 


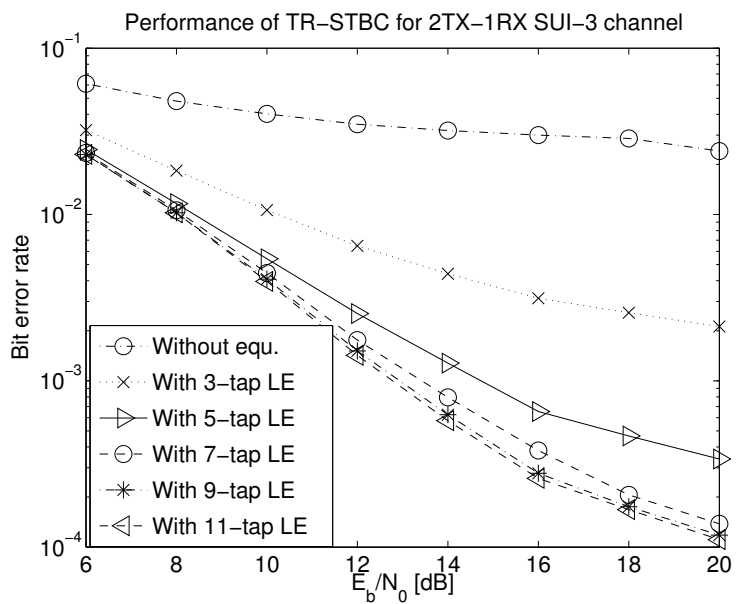

Fig. 8. Performance of TR-STBC for the 2TX-1RX SUI-3 channel in uncoded FWA system.

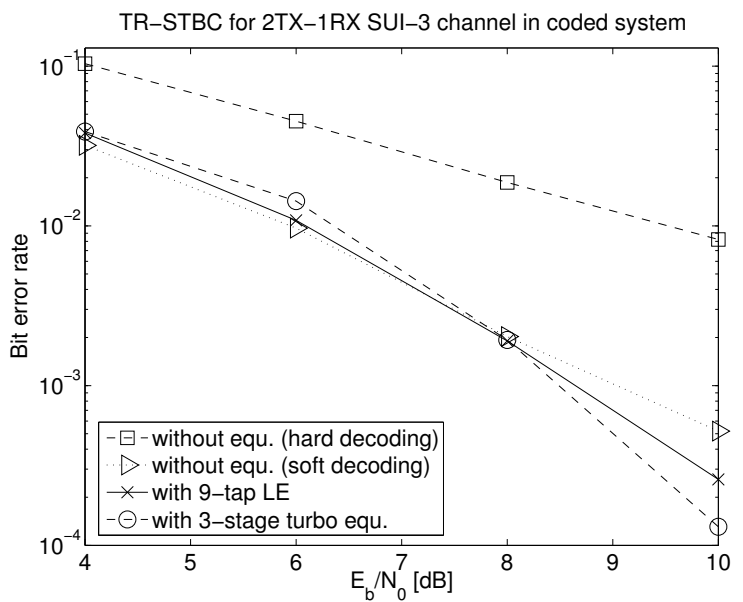

Fig. 9. Performance of TR-STBC for the 2TX-1RX SUI-3 channel in coded FWA system.

schemes (9-tap LE and 3-stage turbo equalization) do not exhibit better performance compared to the case without equalization at low SNR due to the error propagation problem, especially for the turbo equalization scheme which is based on interference cancellation technique. Cancellation using incorrect decision will increase the interference rather than canceling the interference. At high SNR (in this case, when $E_{b} / N_{0} \geq 8 \mathrm{~dB}$ ), the error propagation problem is alleviated, the turbo equalization shows the best performance, however, it is also the most complex among all the schemes. The performance of the 9-tap LE lies somewhere in between, in terms of both performance and complexity.

\section{Conclusions}

In this paper, we apply the TR-STBC scheme for the FWA systems, both with and without channel coding. The TR-STBC converts MIMO multipath channel to SISO multipath channels, and significantly simplifies the receiver design. Our results show that in an uncoded FWA system, ISI presented in each decoupled symbol stream has to be handled by an equalizer; whereas the coded FWA system can operate without an equalizer due to the facts that the TRSTBC exploits both spatial and temporal diversities, and the effect of ISI is mitigated by channel coding. At high 6
SNR, the performance of the coded system can be further improved by employing equalization or turbo equalization schemes at the cost of higher computational complexity.

\section{REFERENCES}

[1] IEEE 802.16 Working Group on Broadband Wireless Access Standards. available at http://grouper.ieee.org/groups/802/16/. 2002.

[2] Draft ETSI TS 101, Broadband Radio Access Networks (BRAN), HIPERMAN. available at http://portal.etsi.org/bran/Summary.asp, 2002.

[3] C. Eklund. "IEEE Standard 802.16: a technical overview of the wirelessMAN air interface for broadband wireless access". IEEE Communications Magazine, pp. 98-107, June, 2002.

[4] H. Bolcskei, A. Paulraj, K. Hari, R. Nabar, W. Lu. "Fixed Broadband Wireless Access: State of the Art, Challenges, and Future Directions". IEEE Communication Magazine, pp. 100-108, Jan. 2001.

[5] W. Webb. "Broadband fixed wireless access as a key component of the future integrated communications environment". IEEE Communications Magazine, pp. 115-121, Sept. 2001.

[6] V. Tarokh, N. Seshadri, A. Calderbank. "Space-time codes for high data rates wireless communications: performance criterion and code construction". IEEE Transactions on Information Theory, vol. 44, pp. 744-765, March 1998.

[7] A. Alamouti. "A simple transmit diversity technqiue for wireless communications". IEEE Journal on Selected Areas in Communications, vol. 16, no. 8, pp. 1451-1458, Oct. 1998.

[8] V. Tarokh, N. Seshadri, A. Calderbank. "Space-time block coding from orthogonal designs". IEEE Transactions on Information Theory, vol. 45, pp. 1456-1467, July 1999.

[9] P. Xiao, R. Carrasco, I. Wassell. "Theoretical Analysis of Single and Multiple antenna FWA Systems". Submitted to IEE Proceedings on Communications.

[10] E. Lindskog, A. Paulraj. "A transmit diversity scheme for channels with intersymbol interference". IEEE International Conference on Communications, pp. 307-311, June 2002.

[11] V. Erceg. "An empirically based path loss model for wireless channels in suburban environments". IEEE JSAC, vol. 17, no. 7, pp. 1205-1211, July 1999.

[12] A. Viterbi. "An intuitive justification and a simplified implementation of the MAP decoder for convolutional codes". IEEE Journals on Selected Areas in Communications, vol. 16, no. 2, pp. 260-264, Feb. 1998.

[13] P. Xiao, R. Carrasco, I. Wassell. "MVU channel estimation for FWA MIMO channels". Submitted to IEEE Globecom 2006. 